\title{
Compósitos Termoplásticos com Madeira
}

\author{
Carlos A. Correa, Carla N.P. Fonseca, Silmara Neves \\ PPGECM, UAACET, Universidade São Francisco \\ Carlos A. Razzino, Elias Hage Jr. \\ Departamento de Engenharia de Materiais, UFSCar
}

\begin{abstract}
Resumo: Dentre muitas fibras naturais de interesse tecnológico, o resíduo de madeira substitui com vantagens as cargas e reforços tradicionalmente empregados em compostos e compósitos poliméricos, particularmente os de origem mineral, tais como talco, $\mathrm{CaCO}_{3}$ e fibra de vidro. A tecnologia dos chamados Wood-Plastic Composites (WPC's) envolve conceitos de compatibilidade e processabilidade e apresenta grandes desafios tecnológicos para a formulação e estabilização da mistura devido à baixa estabilidade térmica da celulose. Muitos dos avanços tecnológicos em WPC's dependem de uma análise criteriosa das características físicas de seus componentes e das condições de processamento do sistema, particularmente aspectos relacionados a sua compatibilização. No presente trabalho são apresentados estudos sobre a reatividade do polipropileno modificado com anidrido maleico (PP-MAH) utilizado como compatibilizante em compósitos termoplásticos de polipropileno com farinha de madeira. A influência da carga celulósica e a eficiência do compatibilizante nas propriedades reológicas, térmicas, mecânicas e morfológicas do sistema foi avaliada através de medidas de rigidez, grau de cristalinidade, temperatura de cristalização e fusão cristalina levando-se em conta as características da resina, a distribuição granulométrica e o teor de umidade da farinha de madeira. Observou-se que os compósitos compatibilizados com PP-MAH apresentaram ganhos significativos de rigidez em relação aos compósitos não-modificados independentemente do tipo de farinha de madeira empregada. As propriedades térmicas do polímero mostraram-se sensíveis à distribuição granulométrica e algumas composições apresentaram tendência ao escurecimento e à elevação da temperatura de cristalização do polímero na presença do reforço celulósico. Imagens obtidas por microscopia eletrônica de varredura ilustram o molhamento da fibra celulósica pela resina termoplástica quando na presença do compatibilizante indicando a possível ocorrência de reações de esterificação na interface polímero-madeira.
\end{abstract}

Palavras-Chave: Compósitos termoplástico-celulósicos, tecnologia polímero-madeira, compatibilização, reometria de torque, análise térmica.

\section{Wood-Plastic Composites}

Abstract: Wood waste fibers (WWF), mostly known as wood flour, can replace mineral fillers such as talc, $\mathrm{CaCO}_{3}$ and fiberglass in the reinforcement of thermoplastics with great advantage. Wood-plastics composites (WPC's) technology includes concepts of compatibility and processing and yet presents technical challenges in grade formulation and stabilisation of the composite system. Owing to thermal constraints in wood flour processing, commodities such as polyolefins (PP, PEAD/PEBD), styrenics (PS e HIPS) and vinyls (PVC) represent the bulk of the thermoplastics used in WPC's applications. In the present work, a brief overview of the latest developments in WPC's processing technology is discussed in the light of technical characteristics and performance of this novel class of thermoplastics composites in emerging applications. Some investigations on compatibilizing efficiency of the polypropylene modified with maleic anhydride (PP-MAH) are presented for polypropylene formulations compounded with different types of pinus wood waste. The influence of the cellulosic filler and the efficiency of compatibilizers on thermal, mechanical and morphology of the composite system was evaluated through stiffness measurements, degree of cristallinity, crystallization and melt temperatures in regard to the resin intrinsic properties and sawdust characteristics. Electron microscopy micrographs illustrate in great detail the filler wetting by the resin in the presence of the maleic anhydride in the polypropylene used as compatibilizer suggesting that sterification reactions may take place at the woodpolymer interface.

Keywords: Thermoplastic composites, wood-plastics technology, compatibilizers, torque rheometer, thermal analysis.

Autor para correspondência: Carlos A. Correa, Universidade São Francisco, Rua Alexandre R. Barbosa 45, CEP: 13521-900, Itatiba, SP. E-mail: carlos.correa@saofrancisco.edu.br 


\section{Introdução}

A preparação de compósitos de madeira com polímeros é uma prática antiga, particularmente quanto ao uso de resinas termofixas como uréia-, fenol-, ou melamina-formaldeído e isocianatos na produção de painéis MDF (medium-density fiberboard). A utilização de farinha ou fibra de madeira como carga em termoplásticos, também já é conhecida desde a década de 70 pela indústria automobilística, que emprega compósitos de polipropileno com farinha de madeira, conhecidos no mercado, como woodstock $^{\circledR}$. Esses compósitos são extrusados e laminados em chapas para revestimento interno de portas e porta-malas de veículos em uso corrente. Durante pelo menos três décadas a tecnologia do woodstock ${ }^{\circledR}$ foi dominada por uns poucos técnicos especialistas não havendo maiores interesses acadêmicos no assunto. Trabalhos de cunho mais acadêmico, acompanhados por um grande número de patentes depositadas por grandes corporações do mercado de construção civil e automobilístico, surgiram nos EUA nos anos 90 relacionando a utilização de resíduos de papel e madeira como cargas para termoplásticos ${ }^{[1-4]}$. Embora a viabilidade técnica para produção de compósitos celulósicos termoplásticos tenha sido comprovada através desses trabalhos, no Brasil ainda se encontra uma certa relutância por parte da indústria de compostos termoplásticos em empregar essa tecnologia. Na maioria dos casos, essa relutância é decorrente da falta de informações técnicas sobre os materiais e os processos, de certificação local de fornecedores de matéria-prima, ausência de equipamentos específicos e desenvolvimentos de novos mercados para esses produtos.

Os estudos mercadológicos realizados nos EUA e na Europa sobre o uso de fibras celulósicas ou farinha de madeira (WWF-wood waste flour) como carga e reforço em termoplásticos revelam que a substituição da madeira convencional por compósitos termoplásticos apresenta-se como alternativa viável ao reaproveitamento de resíduos, com inúmeras vantagens ${ }^{[1,3]}$ :

- Maior resistência à umidade e deterioração ambiental;

- Resistência a pragas e insetos;

- Podem ser extrusados em perfis com formatos diversificados;

- Apresentam melhor estabilidade dimensional;

- Resistência ao empenamento e trincas;

- Possuem menor custo de manutenção de rotina;

- Maior durabilidade em ambientes agressivos como marinas e piscinas;

- São totalmente recicláveis e imitam em aspecto a madeira;

- Dispensam o uso de proteção superficial como tintas e vernizes.

No Brasil, estudos recentes relacionam o uso de resíduos de madeira em inúmeras aplicações ${ }^{[5]}$. Nos EUA e na Europa, já existe uma legislação específica que limita a queima indiscriminada de derivados celulósicos, principalmente papel e madeira, o que acabou incentivando a busca de alternativas para reaproveitamento desses resíduos. Nesses paí- ses, a escassez de madeira também tem provocado o aparecimento de produtos conhecidos como compósitos termoplásticos com madeira ou wood-plastic composites, WPC. Esses produtos têm encontrado grande aceitação no mercado em aplicações como perfis para construção civil e como componentes automobilísticos graças a sua leveza, versatilidade e baixo custo, quando comparados com a madeira in natura ou outros compósitos poliméricos reforçados com cargas minerais. Ainda nos EUA, o mercado para termoplásticos celulósicos com aplicação em materiais de construção apresentou um crescimento vertiginoso na última década e vem crescendo a uma taxa de $15 \%$ ao ano. Esse fato pode ser comprovado pelo aumento na demanda dessas matérias-primas para uso como carga em termoplásticos e pelo aumento no número de patentes depositadas nos EUA relacionadas ao tema conforme dados apresentado nas Figuras 1 e 2 .

A maior fatia do mercado de WPC's ainda está na construção civil com materiais do tipo polietilenos, polipropilenos e PVC extrusados com resíduo de madeira na forma de perfis para pisos e assoalhos, batentes de janelas e portas, revestimentos dentre muitas outras aplicações. Os principais mercados para WPC's por aplicação final do produto são mostrados na Figura 3. Estes dados foram apresentados na última conferência sobre compósitos termoplásticos com madeira realizada em Baltimore nos EUA (2000) com inúmeros trabalhos destacando a importância e o interesse no setor

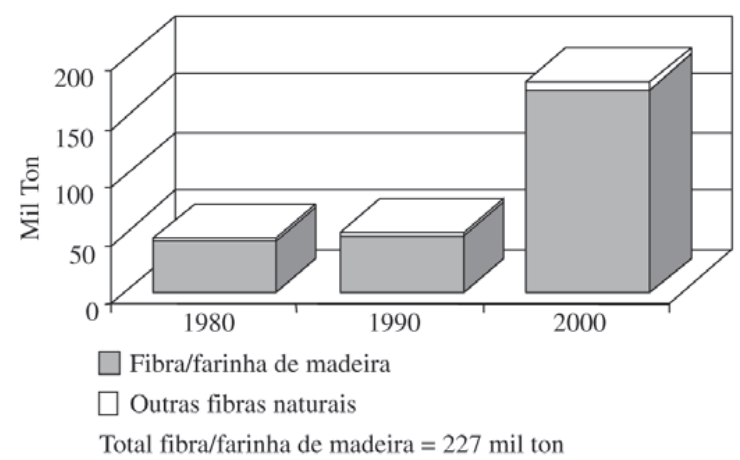

Figura 1. Crescimento na demanda por fibras naturais para uso em compósitos nos EUA 2000[1].

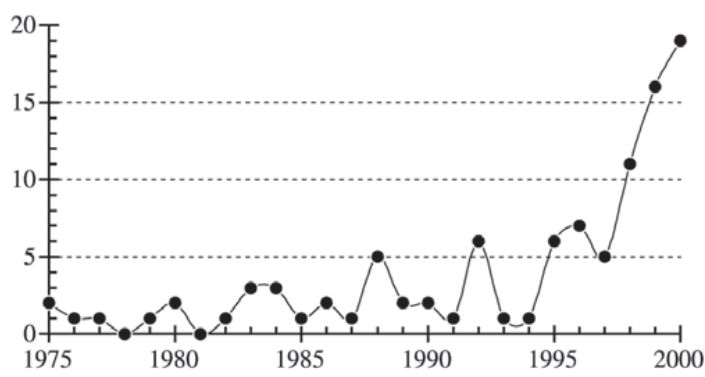

Figura 2. Crescimento no número de patentes depositadas nos EUA de 1975 a 2000 relacionadas com materiais e processos de compósitos termoplásticos com madeira ${ }^{[2]}$. 


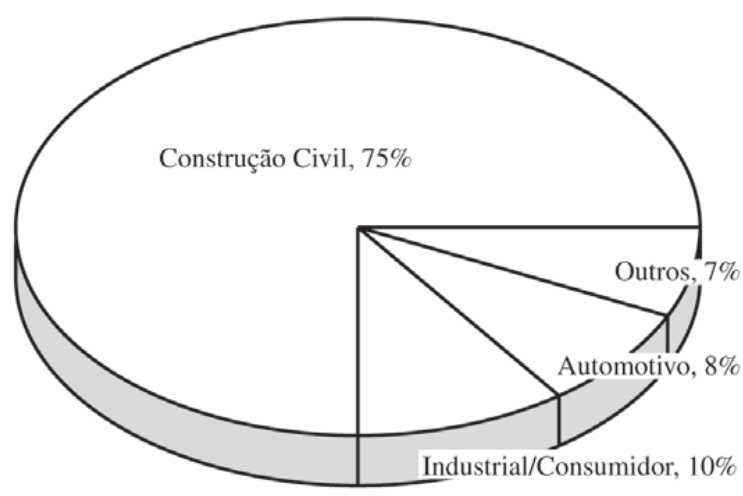

Figura 3. Principais mercados para compósitos termoplásticos com madeira por aplicação final do produto ${ }^{[1]}$.

a partir da última década.

\section{Avanços tecnológicos no processamento de WPC's}

Inúmeros aspectos devem ser observados no processamento de termoplásticos com resíduos de madeira. A umidade e a granulometria devem ser rigidamente controladas, uma vez que esta produz descontinuidades de processo e peças com características inaceitáveis devido à presença de bolhas ou manchas superficiais causadas por processos termo-oxidativos ${ }^{[6,7]}$. Assim, como principal requisito, o resíduo celulósico deve ser pré-secado e zonas de degasagem devem ser utilizadas para remoção da umidade residual durante o processamento. A baixa temperatura de degradação da celulose na faixa de 200 a $220{ }^{\circ} \mathrm{C}$ constitui um fator limitante do processo, exceto quando os tempos de residência são minimizados. A exposição do resíduo de madeira a temperaturas acima dessa faixa libera voláteis, provoca descoloração, aparecimento de odor e a fragilização do compósito ${ }^{[3,4]}$. Isso tem restringido o uso de termoplásticos em WPC's às principais resinas comerciais como poliolefinas, plásticos estirênicos e o PVC.

Além das propriedades intrínsecas dos componentes do sistema, o desempenho de WPC's é fortemente dependente do processo de manufatura. Os procedimentos tradicionalmente utilizados para preparação de concentrados ou masterbatches a base de compósitos celulósicos termoplásticos são processos do tipo batelada em misturador intensivo do tipo k-mixer ou processo contínuo em extrusora de dupla rosca conforme ilustrado nas Figuras 4 e 5 respectivamente. Em ambos os casos o compósito polímero-madeira obtido pode ser granulado e processado em extrusora de rosca única para obtenção de perfis e chapas laminadas.

Com base nessas peculiaridades, existe atualmente uma grande discussão entre os maiores fabricantes de equipamentos tais como Bauzzano, Davis-Standard, Leistritz, Millacron Inc., Krupp Werner \& Pfleiderer, etc., sobre a melhor configuração de máquina para processamento de compósitos termoplásticos com madeira. Estes equipamentos podem ser classificados em quatro categorias distintas ${ }^{[8,9]}$.

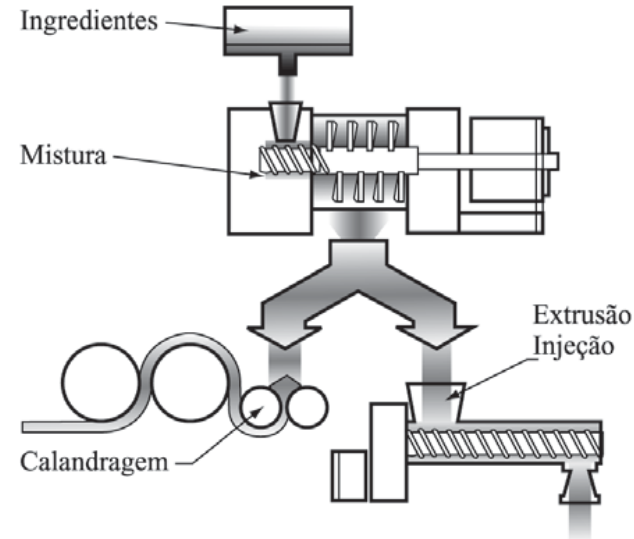

Figura 4. Processo por batelada em misturador interno tipo k-mixer. (Catálogo técnico Drais $\left.{ }^{\circledR}\right)$.

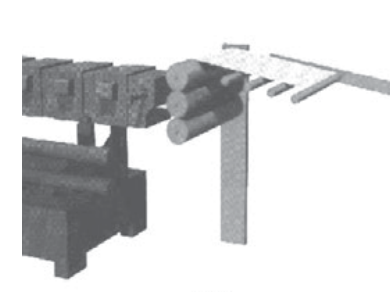

(a)

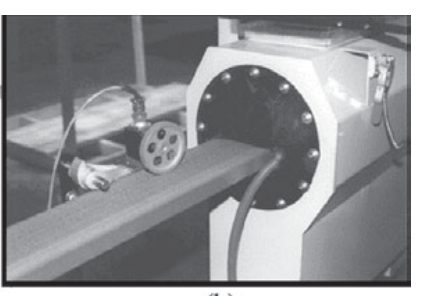

(b)
Figura 5. Processos de extrusão contínua de termoplásticos com resíduos de madeira. (a) Laminados obtidos por extrusão direta para uso em painéis automobilísticos (processo woodstock $^{\circledR}$ ) e (b) Extrusão de perfis acabados para uso em aplicações estruturais como plastic lumber na construção civil.

- A - Madeira pré-secada; mistura pré-homogeneizada (Pre-dry; Pre-mix): Nesse processo são utilizados equipamentos no qual a fibra de madeira é submetida a processos de pré-secagem em níveis de umidade inferiores a $1 \%$ e alimentada em uma extrusora rosca-dupla contra-rotacional juntamente com o polímero, normalmente na forma de pó. A mistura polímero-madeira e aditivos é preparada em misturadores intensivos do tipo Henschel antes de ser alimentada na extrusora. Esse sistema é muito utilizado para processamento de termoplásticos com baixa estabilidade térmica como o PVC.

- B - Madeira pré-secada; alimentação polímero-madeira em separado (Pre-dry; Split Feed): Nesse processo a resina e a fibra são alimentadas em separado propiciando um melhor controle do tempo de residência da carga celulósica durante o processamento. São normalmente utilizadas extrusoras de rosca-dupla de grande capacidade, com portos laterais de alimentação, onde a fibra é misturada ao polímero fundido, passando por zonas de mistura distributiva e desgaseificação da umidade residual.

- C- Madeira úmida primeiro; alimentação do polímero fundido (Wood First; Melt Feed): Nesse processo são necessárias duas extrusoras que operam simultaneamente; uma extrusora primária secando a farinha de 
madeira e uma outra menor plastificando o polímero e os aditivos. A empresa Davis-Standard dos EUA patenteou um equipamento dedicado para o processamento de compósitos termoplásticos com essas características, denominado Woodtruder ${ }^{\circledR}$ com capacidade de até $1000 \mathrm{lbs} / \mathrm{h}$.

- $D$ - Madeira úmida primeiro; alimentação em separado (Wood First, Split Feed): Nesse processo a farinha de madeira pode ser alimentada ainda úmida na zona de alimentação e a mistura de resina e aditivos introduzida posteriormente no barril através de um alimentador lateral. Todavia esse processo requer normalmente equipamentos com barris muito longos (L/D 44 ou 48:1) e com zonas de desgaseificação próximas a zona de alimentação para remoção da umidade da madeira, o que nem sempre é possível.

Nos processos nos quais a extrusora é utilizada na secagem da farinha de madeira, pode haver problemas de consistência da massa fundida devido a alterações de umidade. Os custos associados a essa prática ainda têm sido objeto de calorosas discussões entre fabricantes de equipamentos e processadores. Dessa forma, a alternativa mais segura ainda tem sido a utilização de madeira pré-secada adquirida junto aos fornecedores de farinha/fibra de madeira.

Associada às propriedades intrínsecas dos componentes da mistura e as condições de processamento, a compatibilização interfacial dos WPC's é um dos requisitos mais importantes no desenvolvimento dessa classe de compósitos poliméricos. Na sequência são apresentados resultados de alguns estudos relacionados à compatibilização de polipropileno e farinha de madeira utilizando como agente de acoplamento um copolímero proveniente da reação com anidrido maleico.

\section{Estudos de compatibilização de compósitos termoplásticos com madeira}

O efeito da incorporação de fibras naturais lignocelulósicas, como kenaf, sisal, juta, algodão e madeira nas propriedades do polipropileno tem sido objeto de estudos extensivos. Considerações sobre processo, compatibilização e propriedades têm sido abordadas por inúmeros autores ${ }^{[10-17]}$. Esse amplo interesse baseia-se no fato de que a incorporação de fibras naturais em termoplásticos poliolefínicos apolares, o caráter polar da celulose requer a compatibilização do sistema através da incorporação dos chamados agentes de acoplamento ou de adesão interfacial. A escolha de agentes compatibilizantes para promover uma melhor adesão interfacial entre o reforço celulósico e a matriz polimérica poliolefínica deve seguir alguns critérios básicos: Inicialmente o compatibilizante ou agente de acoplamento, em contato com a superfície do reforço deve interagir fortemente com as fibras através de ligações covalentes fortes ou interações secundárias do tipo ácido-base ou pontes de hidrogênio. Isto implica que uma quantidade suficiente de grupos funcionais deve estar presente no compatibilizante que possibilite a reação com as hidroxilas da celulose. Outro aspecto considerado é o comprimento das cadeias do compatibilizante que deve possuir cadeias suficientemente longas de alta massa molecular que permita a formação de emaranhamentos moleculares com a matriz polimérica na interfase através de ancoramento mecânico. Alguns autores relacionam o tratamento superficial da fibra como forma de optimização do processo de compatibilização ${ }^{[15-20]}$.

Do ponto de vista mecânico, os agentes compatibilizantes atuam no sentido de evitar o processo de delaminação da fibra na matriz polimérica que ocorre por incompatibilidade química e acaba comprometendo o reforçamento do compósito devido a transferência ineficiente de esforços na interface fibra-matriz. A formação de ligações covalentes através de reações de esterificação e interações secundárias por pontes de hidrogênio entre o anidrido maleico do PPMAH e as hidroxilas da celulose foram observadas por FTIR e ESCA tal como ilustrado na Figura 6 para a o PP-MAH ${ }^{[4,10-11]}$.

Acredita-se que o compatibilizante ideal para polipropileno reforçado com fibra celulósica deveria ser um copolímero de polipropileno-anidrido maleico (PP-MAH) contendo alto teor de MAH e viscosidade controlada através do tamanho e distribuição de tamanhos de cadeia. Teores de 0,5\% em massa de MAH no PP-MAH têm sido considerados como suficientes para promover melhoras nas propriedades mecânicas em compósitos de PP-Kenaf. Em polipropileno com farinha de madeira esses valores situam-se entre 6 a $10 \%$ em massa ${ }^{[10]}$. Outra consideração importante para a eficiência de reforçamento consiste na geração de zonas de trans-cristalinidade na interfase que depende do teor de MAH no compatibilizante e determina as condições de co-cristalização entre o PP e PP-MAH. Aspectos conflitantes sobre as condições de ocorrência de transcristalinidade nesses sistemas ainda exigem pesquisas mais aprofundadas ${ }^{[9,10,22]}$.

Além dos aspectos químicos ligados a compatibilização da fibra com a matriz, o desempenho do compósito ainda

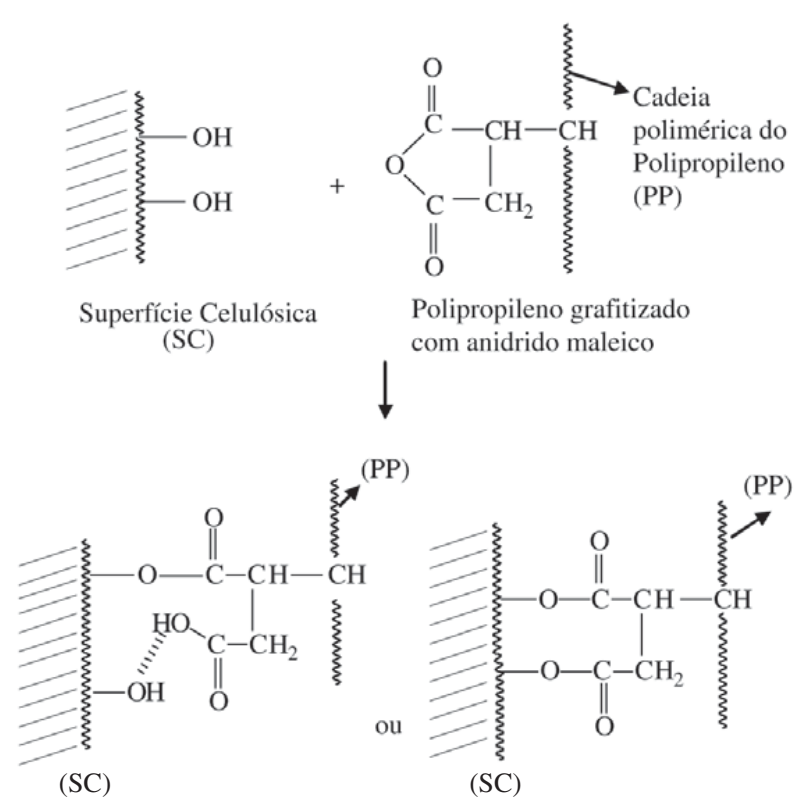

Figura 6. Provável reação de esterificação e interações por pontes de hidrogênio da celulose com o polipropileno-g-anidrido maleico (PP-MAH) ${ }^{[4,10 \sim 17]}$. 
depende das características do reforço, ou seja, distribuição granulométrica ou razão de aspecto; tamanho e distribuição de tamanho de partículas; área superficial e natureza química da superfície e a fração volumétrica máxima de empacotamento da carga ${ }^{[26]}$. No presente trabalho foram realizados alguns estudos reológicos para avaliação da compatibilização do polipropileno com farinha de madeira utilizando como compatibilizante um copolímero de polipropileno com anidrido maleico. Ensaios mecânicos e análises térmicas por calorimetria diferencial de varredura foram utilizados para avaliação da eficiência do reforçamento e da estabilidade térmica do sistema. Os compósitos utilizados no estudo foram preparados através de extrusão direta em extrusora dupla-rosca utilizando o método de madeira pré-secada/mistura pré-homogeneizada (pre-dry/pre-mix).

\section{Experimental}

\section{Materiais}

Neste trabalho foi utilizado um polipropileno homopolímero (PP-Ho) com índice de fluidez de 0,8 g/10 min, determinado pelo método ASTM D-1238 (190 $\left.{ }^{\circ} \mathrm{C} / 5 \mathrm{~kg}\right)$. Foi testado como agente compatibilizante um polipropileno modificado com anidrido maleico (PP-MAH), fornecido pela empresa Uniroyal sob a marca Polybond ${ }^{\circledR}$. As porcentagens de MAH enxertado no PP segundo o fabricante situa-se na faixa de 5 a $6 \%$. O índice de fluidez do PP-MAH, medido nas condições $230{ }^{\circ} \mathrm{C} / 2,16 \mathrm{~kg}$ foi de 7,0 g/10 min.

Na preparação dos compósitos, foram utilizados três tipos de resíduos de madeira pinus na forma de farinha de madeira $(W W F)$ : a) farinha de madeira grau comercial, PinhopóMesh\#45 (WWF-A); b) a serragem de pinus caribea resíduo da manufatura de lápis na empresa Faber-Castell S/A (WWF-B); c) Resíduo de pinus obtido em madeireiras da região de Itatiba-SP (WWF-C). A densidade aparente do resíduo de madeira WWF-A foi fornecida como $0,250 \mathrm{~g} / \mathrm{cm}^{3}$ e as demais composições não foram obtidas. O teor de umidade final da madeira utilizada foi medido através da relação peso seco/peso úmido após secagem em estufa a $100{ }^{\circ} \mathrm{C}$ por 48 h, ficando em cerca de $8 \%$. A composição das amostras dos compósitos de PP-Ho/PP-MAH/WWF preparadas para o presente estudo são apresentadas na Tabela 1 .

As distribuições granulométricas das amostras de farinha de madeira foram obtidas através do método de pesagem de massa residual ou porcentagem retida em peneiras padronizadas ASTM conforme ilustrado na Tabela 2. As condições de ensaio foram massa inicial de $100 \mathrm{~g}$ e tempo de peneiramento de $30 \mathrm{~min}$. As características morfológicas das amostras são ilustradas na Figura 7.

\section{Metodologia}

Um resumo das formulações, procedimentos e análises realizadas estão resumidos no fluxograma da Figura 8.

\section{Reometria de torque}

Análise da compatibilização reativa in situ do polipropileno com a farinha de madeira foram realizados em um reômetro de torque Haake Polylab System, mod. Rheocord 300 p/Rheomix 600 p. Os materiais foram pesados nas proporções relativas a cada formulação e alimentados na câmara a $190{ }^{\circ} \mathrm{C}$, por $10 \mathrm{~min}$ sob rotação de $60 \mathrm{rpm}$. Foram obtidos gráficos de torque em função do tempo e temperatura da massa e os dados foram tabelados.

\section{Preparação dos compósitos}

Após a moagem e classificação granulométrica, a farinha de madeira foi tratada com lubrificantes e uma mistura de óleos minerais em um misturador de alta intensidade do tipo Henschel. A etapa seguinte de incorporação da farinha de madeira ao termoplástico foi realizada em uma extrusora de

Tabela 1. Grade de amostras

\begin{tabular}{|c|c|c|c|c|c|c|}
\hline \multirow{2}{*}{$\begin{array}{l}\text { Materiais / } \\
\text { Formulações }\end{array}$} & \multirow{2}{*}{$\begin{array}{c}\text { PP-Ho } \\
\% \text { massa }\end{array}$} & \multirow{2}{*}{$\begin{array}{c}\text { PP-MAH } \\
\% \text { massa }\end{array}$} & \multicolumn{3}{|c|}{ WWF \% massa } & \multirow[t]{2}{*}{ OBS. } \\
\hline & & & $\mathbf{A}$ & B & $\mathbf{C}$ & \\
\hline PP-Ho & 100 & - & - & - & - & Puro \\
\hline \multirow{3}{*}{ PP-MAH } & - & 100 & - & - & - & Não-compatibilizado \\
\hline & 70 & - & 30 & - & - & \\
\hline & 70 & - & - & - & 30 & \\
\hline \multirow[b]{2}{*}{ PP/PP-MAH/WWF } & 60 & 10 & 30 & - & - & \\
\hline & 60 & 10 & - & - & 30 & \\
\hline PP-Ho/ WWF & 50 & - & - & 50 & - & Não-compatibilizado \\
\hline \multirow{3}{*}{ PP-Ho/PP-MAH/WWF } & 49 & 1 & - & 50 & - & \\
\hline & 45 & 5 & - & 50 & - & \\
\hline & 40 & 10 & - & 50 & - & \\
\hline \multirow{5}{*}{ PP-MAH/WWF } & - & 90 & - & - & 10 & \\
\hline & - & 80 & - & - & 20 & \\
\hline & - & 70 & - & - & 30 & \\
\hline & - & 60 & - & - & 40 & \\
\hline & - & 50 & - & - & 50 & \\
\hline
\end{tabular}


dupla-rosca co-rotacional de bancada B\&P Process Equipments Systems, no qual o polipropileno, a farinha de madeira e o compatibilizante foram alimentados diretamente no funil da extrusora pelo método tradicional de Madeira présecada/mistura pré-homogeneizada (Pre-dry/Pre-mix). O perfil de temperaturas utilizado ao longo de barril foi 170 a $190{ }^{\circ} \mathrm{C}$ com uma velocidade de rotação de rosca de 60 a 100 rpm.

\section{Moldagem de corpos de prova por injeção}

As propriedades mecânicas foram avaliadas através do

Tabela 2. Classificação granulométrica das amostras de farinha de madeira, método das peneiras.

\begin{tabular}{lccc}
\hline Malha ASTM & $\begin{array}{c}\text { WWF-A } \\
\text { \%Retida }\end{array}$ & $\begin{array}{c}\text { WWF-B } \\
\text { \% Retida }\end{array}$ & $\begin{array}{c}\text { WWF-C } \\
\text { \% Retida }\end{array}$ \\
\hline $30(590 \mu \mathrm{m})$ & 1,5 & 31,7 & 42,5 \\
$40(420 \mu \mathrm{m})$ & 36 & 42,3 & 23,2 \\
$60(250 \mu \mathrm{m})$ & 42,5 & 10,6 & 14,8 \\
$100(150 \mu \mathrm{m})$ & 16,2 & 5,3 & 11,9 \\
Fundo & 3,8 & 10,2 & 7,6 \\
\hline
\end{tabular}

ensaio de tração uniaxial com base na norma ASTM D-638 utilizando corpos de prova tipo M-I e barras para ensaio IZOD com base na norma ASTM D-256. Os corpos de prova foram moldados por injeção em uma injetora com força de fechamento de 30 tons, modelo $270 \mathrm{~V}$, marca ARBURG.

\section{Caracterização física}

Os ensaios foram realizados segundo a norma ASTM D-638 em atmosfera padrão a $23^{\circ} \mathrm{C}$ e $65 \%$ de UR. Para avaliar o efeito da umidade nas propriedades físicas do compósito, algumas amostras foram mantidas em dessecador após a moldagem e ensaiadas a sêco como moldadas e outras foram imersas em água por $48 \mathrm{~h}$ antes dos ensaios. A velocidade de ensaio foi de 5,0 $\mathrm{mm} / \mathrm{min}$. Ensaios de impacto foram realizados à temperatura ambiente em corpos de prova tipo IZOD entalhados.

\section{Caracterização térmica}

As análises térmicas das amostras de polipropileno com madeira compatibilizados e não-compatibilizados foram realizados em um calorímetro (NETZSCH-DSC 204) sob uma
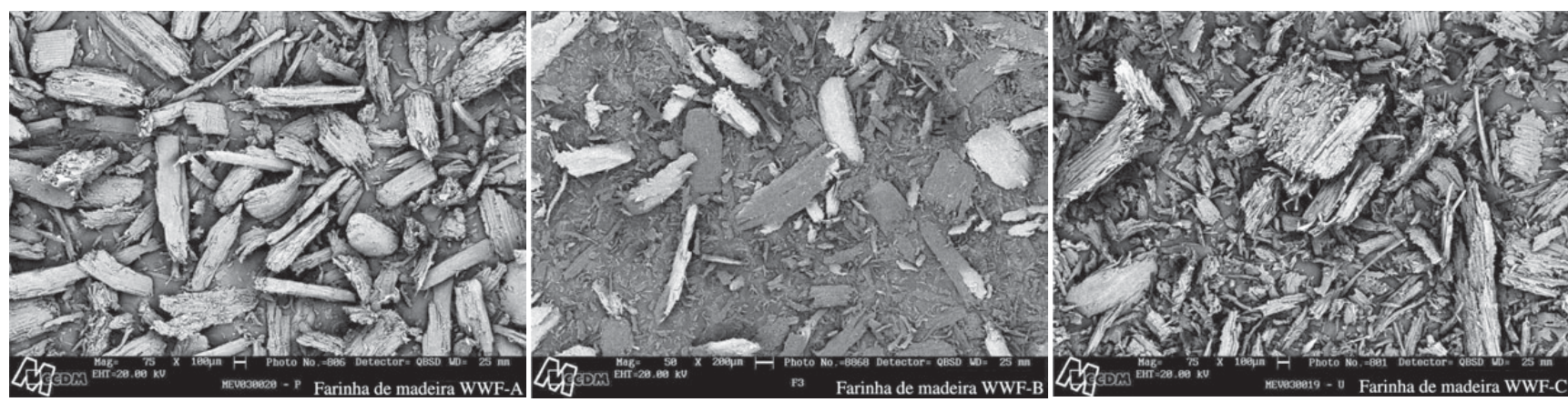

Figura 7. Aspectos característicos das amostras de farinha de madeira obtidas por microscopia eletrônica de varredura.



Figura 8. Sumário das formulações, etapas de preparação dos compósitos e análises realizadas. 
Tabela 3. Efeito da concentração de PP-MAH na mistura 50PP-Ho/50WWF-B.

\begin{tabular}{lcccc}
\hline \multicolumn{1}{c}{ Material } & $\begin{array}{c}\text { Tempo } \\
(\mathbf{m i n})\end{array}$ & $\begin{array}{c}\text { Torque } \\
(\mathbf{N . m})\end{array}$ & $\begin{array}{c}\text { Temperatura da massa } \\
\left({ }^{\circ} \mathbf{C}\right)\end{array}$ & $\begin{array}{c}\text { Velocidade } \\
(\mathbf{R P M})\end{array}$ \\
\hline PP-Ho & 10 & 9,2 & 212 & 60 \\
50PP-Ho/50WWF-B & 10 & 6,0 & 208 & 60 \\
49PP-Ho/1PP-MAH/50WWF-B & 10 & 5,2 & 207 & 60 \\
45PP-Ho/5PP-MAH/50WWF-B & 10 & 4,4 & 207 & 60 \\
40PP-Ho/10PP-MAH/50WWF-B & 10 & 5,5 & 208 & 60 \\
\hline
\end{tabular}

Tabela 4. Efeito da concentração da farinha de madeira WWF-C na reologia do PP-MAH.

\begin{tabular}{lcccc}
\hline \multicolumn{1}{c}{ Material } & $\begin{array}{c}\text { Tempo } \\
(\mathbf{m i n})\end{array}$ & $\begin{array}{c}\text { Torque } \\
(\mathbf{N . m})\end{array}$ & $\begin{array}{c}\text { Temperatura da massa } \\
\left({ }^{\circ} \mathbf{C}\right)\end{array}$ & $\begin{array}{c}\text { Velocidade } \\
(\mathbf{R P M})\end{array}$ \\
\hline PP-MAH & 10 & 4,6 & 204 & 60 \\
50PP-Ho/50WWF-C & 10 & 7,1 & 208 & 60 \\
50PP-MAH/50WWF-C & 10 & 3,8 & 201 & 60 \\
60PP-MAH/40WWF-C & 10 & 3,5 & 205 & 60 \\
70PP-MAH/30WWF-C & 10 & 4,0 & 205 & 60 \\
80PP-MAH/20WWF-C & 10 & 5,9 & 206 & 60 \\
90PP-MAH/10WWF-C & 10 & 4,7 & 202 & 60 \\
\hline
\end{tabular}

atmosfera de $\mathrm{N}_{2}$ a uma taxa de aquecimento de $20^{\circ} \mathrm{C} / \mathrm{min}$ na faixa de -50 a $300{ }^{\circ} \mathrm{C}$. Foram considerados apenas os dados de fusão e cristalização da segunda varredura que foram obtidos em duplicata. A porcentagem de cristalinidade $(\% \mathrm{Xc})$ foi calculada utilizando-se o valor de referência $210 \mathrm{~J} / \mathrm{g}$ da entalpia de fusão da amostra $100 \%$ cristalina obtido na literatura ${ }^{[7]}$.

\section{Caracterização morfológica}

Os estudos morfológicos foram realizados em amostras fraturadas de corpos de prova testados em tração utilizando um microscópio eletrônico de varredura modelo LEO 400 operado com uma voltagem de aceleração de 20 kV. As imagens foram obtidas a partir da optimização dos sinais de elétrons secundários e retroespalhados.

\section{Resultados e Discussão}

\section{Caracterização reológica dos compósitos}

A reometria de torque permite estimar a viscosidade de polímeros fundidos de forma indireta através do torque necessário para se manter uma velocidade constante com os rotores do reômetro, a uma dada temperatura. Portanto é possível monitorar in situ processos termo-oxidativos ou reações de reticulação, que estão diretamente relacionadas a alterações na viscosidade do polímero. Estas reações podem ser a esterificação da celulose com o anidrido maleico (aumento de viscosidade) ou reações de degradação da resina termoplástica (redução da viscosidade). No presente caso, foram observadas alterações de viscosidade em função do tipo e concentração da farinha de madeira, da resina e do compatibilizante. Os resultados obtidos para misturas 50PP-Ho/50WWF com a farinha de madeira WWF-B e quantidades de 1, 5 e 10\% de PP-MAH são apresentados resumidamente na Tabela 3. O efeito da concentração de farinha de madeira na compatibilização da mistura PP-MAH/WWF foi avaliada variando-se a concentração da farinha WWF-C na faixa de 10 a $50 \%$ conforme dados apresentados na Tabela 4.

Os dados apresentados na Tabela 3 mostram que, tanto o aumento da concentração de farinha de madeira como a do compatibilizante, produzem redução na viscosidade da mistura quando comparados ao polipropileno puro nas mesmas condições de ensaio. Além disso, todas as misturas contendo o PP-MAH apresentaram uma menor viscosidade em relação à mistura não-compatibilizada 50PP/50WWF. Esse fato pode ser explicado em parte pela menor viscosidade do PPMAH em relação ao PP-Ho, embora contrarie a expectativa de que a reação de esterificação entre os grupamentos hidroxila da celulose e o anidrido maleico no polipropileno deveria produzir um aumento na viscosidade do sistema compatibilizado.

Nas misturas PP-MAH/WWF-C apresentadas na Tabela 4, mesmo trabalhando-se com excesso de PP-MAH na mistura, não foi possível se estabelecer uma tendência clara entre alterações da viscosidade do sistema e o aumento da concentração de PP-MAH na mistura.

\section{Características mecânicas dos compósitos PP/WWF}

Em se tratando de produtos com aplicações estruturais, as propriedades mecânicas dos compósitos termoplásticos com madeira são de fundamental importância. Todavia, embora a fibra de madeira apresente módulo de elasticidade em torno de 20-40 GPa, em se tratando de rigidez, as propriedades mecânicas dos compósitos termoplásticos reforçados com farinha de madeira tendem a ser inferiores às da madeira in natura. Todavia, ganhos de rigidez obtidos com a farinha de madeira em relação aos termoplásticos não-modificados tornam-se significativos. Assim, o principal ganho de propriedades dos termoplásticos reforçados com fibra ou farinha de madeira consiste na melhora da rigidez, com maior temperatura de uso e com baixo peso específico, princi- 
palmente se comparados a compósitos similares reforçados com cargas minerais ${ }^{[3,7,24-26]}$.

Normalmente, em compósitos termoplásticos de polipropileno com farinha ou fibra de madeira, a adição de $20 \%$ em massa do reforço celulósico, produz um acréscimo de $30 \%$ no módulo em flexão do compósito enquanto a mesma quantidade em massa de talco eleva a rigidez em cerca de $80 \%$. No entanto, é importante salientar que em compósitos, para efeito de cálculos estruturais, considera-se normalmente a rigidez específica, ou seja, a razão entre o módulo do material e o seu peso específico. Considerando que a densidade aparente do talco é cerca de 11 vezes a densidade da farinha de madeira $\left(2,75 v s .0,2504 \mathrm{~g} / \mathrm{cm}^{3}\right)$, a rigidez específica do compósito termoplástico de polipropileno com farinha de madeira é muito mais atraente do ponto de vista econômico e tecnológico pelas razões já mencionadas. O mesmo raciocínio aplica-se para compósitos de fibras minerais, como a fibra de vidro que apresenta uma densidade da mesma ordem de grandeza do talco.

A Tabela 5 apresenta as propriedades mecânicas da matriz e dos compósitos de polipropileno contendo $50 \%$ de WWF-B sujeitos ou não a imersão em água por $48 \mathrm{~h}$.

Os resultados apresentados na Tabela 5 indicam que a imersão pouco afetou as propriedades dos compósitos e que estes apresentaram maior módulo e menor deformação no escoamento e resistência do que a matriz. Assim, mesmo após a imersão em água, prevaleceu o caráter hidrofóbico da resina no compósito.

Nas misturas em que se adicionou o compatibilizante PPMAH observou-se claramente o aumento da rigidez e da resistência mecânica para os dois resíduos de madeira analisados em comparação com as amostras não compatibilizadas (Tabela 6). Para a farinha de madeira com alto teor de partículas finas, i.e. aproximadamente $7,6 \%$ da massa retida no fundo da peneira (Amostra WWF-C), o ganho não foi tão signifi- cativo como no caso da WWF-A (3,8\% de massa retida), e foi ainda observada uma maior tendência ao escurecimento característico dos processos de degradação térmica provavelmente devido a maior quantidade de partículas finas (fundo da peneira) presentes nas amostras WWF-B e WWF-C.

Os aumentos de resistência à tração observados nas amostras compatibilizadas indicam uma melhor transferência de tensão da matriz para o reforço celulósico na interface decorrente de uma provável ocorrência de ancoramento mecânico ou interações químicas entre os grupos anidrido e as hidroxilas da celulose na interface polímero-madeira.

Os resultados dos testes de impacto apresentados na Tabela 7 ilustram dois aspectos relevantes observados com a adição da farinha de madeira ao polipropileno quando o compósito é compatibilizado e não-compatibilizado. Independente da granulometria da farinha de madeira, a tenacidade do material à fratura aumenta com a incorporação de $30 \%$ de farinha embora com a adição do compatibilizante, a tenacidade é reduzida nos dois casos. A resistência ao impacto em compósitos termoplásticos depende em parte da capacidade da matriz dissipar energia deformacional à altas taxas e as características da interface são determinantes nesse processo. Uma possível explicação para a redução da tenacidade nas amostras compatibilizadas poderia ser uma maior dificuldade de delaminação da fibra na matriz que constitui um mecanismo importante de dissipação de energia.

\section{Comportamento Térmico}

Na Figura 9 são observadas alterações na temperatura de cristalização do polipropileno na presença de dois tipos de madeira (WWF-A e WWF-C) para misturas compatibilizadas e não-compatibilizadas. Essas análises indicaram aumentos de cerca de 2 a $5{ }^{\circ} \mathrm{C}$ na temperatura do pico de cristalização da resina nos compósitos. Estudos recentes,

Tabela 5. Resistência mecânica sob tração de amostras não compatibilizadas de polipropileno com 50\% WWF testadas a seco e após imersão em água por 48h.

\begin{tabular}{lccc}
\multicolumn{1}{c}{ Propriedade } & PP-Ho & $\begin{array}{c}\text { PP-Ho/50WWF-B } \\
\text { (seco) }\end{array}$ & $\begin{array}{c}\text { PP-Ho/50WWF-B } \\
(\mathbf{4 8} \text { h imersão) }\end{array}$ \\
\hline Tensão de escoamento (MPa) & $32,8 \pm 0,6$ & $24,7 \pm 1,41$ & $24,0 \pm 0,3$ \\
Deformação no escoamento (\%) & $14,9 \pm 0,3$ & $2,0 \pm 0,2$ & $1,9 \pm 0,1$ \\
Módulo (GPa) & $1,7 \pm 0,1$ & $3,3 \pm 0,2$ & $3,0 \pm 0,2$ \\
Tensão na Ruptura (MPa)* & - & $24,4 \pm 1,5$ & $23,1 \pm 0,3$ \\
Deformação na ruptura (\%)* & - & $2,2 \pm 0,2$ & $2,1 \pm 0,1$ \\
\hline
\end{tabular}

*Não rompe no limite de extensão das garras do equipamento de teste.

Tabela 6. Efeito do compatibilizante PP-MAH nas propriedades de tração da mistura 70PP/30WWF para dois tipos de madeira testados.

\begin{tabular}{lccc}
\hline \multicolumn{1}{c}{ Propriedade } & $\begin{array}{c}\text { 70PP-Ho / } \\
\text { 30WWF-A }\end{array}$ & $\begin{array}{c}\text { 60PP-Ho/ } \\
\text { 10PP-MAH/ } \\
\text { 30WWF-A }\end{array}$ & $\begin{array}{c}\text { 70PP-Ho / } \\
\text { 30WWF-C }\end{array}$ \\
\hline Tensão de escoamento (MPa) & $30,3 \pm 0,3$ & $35,2 \pm 0,4$ & $32,7 \pm 0,9$ \\
30WWF-C & $33,5 \pm 0,3$ \\
Deformação no escoamento (\%) & $5,8 \pm 0,2$ & $5,1 \pm 0,2$ & $5,9 \pm 0,3$ \\
Módulo (GPa) & $2,6 \pm 0,2$ & $2,9 \pm 0,1$ & $2,6 \pm 0,2$ \\
Tensão na Ruptura (MPa) & $24,6 \pm 1,9$ & $29,5 \pm 0,9$ & $2,7 \pm 0,7 \pm 1,0$ \\
Deformação na ruptura (\%) & $8,0 \pm 0,5$ & $6,3 \pm 0,9$ & $28,3 \pm 1,6$ \\
\hline
\end{tabular}


Tabela 7. Efeito do compatibilizante PP-MAH nas propriedades de impacto da mistura 70PP/30WWF para dois tipos de madeira testados.

\begin{tabular}{|c|c|c|c|c|c|}
\hline & $\overline{\text { PP-Ho }}$ & $\begin{array}{l}\text { 70PP-Ho/ } \\
\text { 30WW-A }\end{array}$ & $\begin{array}{c}\text { 60PP-Ho/ } \\
\text { 10PP-MAH/ } \\
\text { 30WWF-A }\end{array}$ & $\begin{array}{l}\text { 70PP-Ho/ } \\
\text { 30WW-C }\end{array}$ & $\begin{array}{c}\text { 60PP-Ho/ } \\
\text { 10PP-MAH/ } \\
\text { 30WWF-C }\end{array}$ \\
\hline Resistência ao $\operatorname{Impacto}(\mathrm{J} / \mathrm{m})$ & $31,5 \pm 4,1$ & $43,6 \pm 2,1$ & $36,3 \pm 4,1$ & $37,8 \pm 2,0$ & $32,9 \pm 2,2$ \\
\hline
\end{tabular}

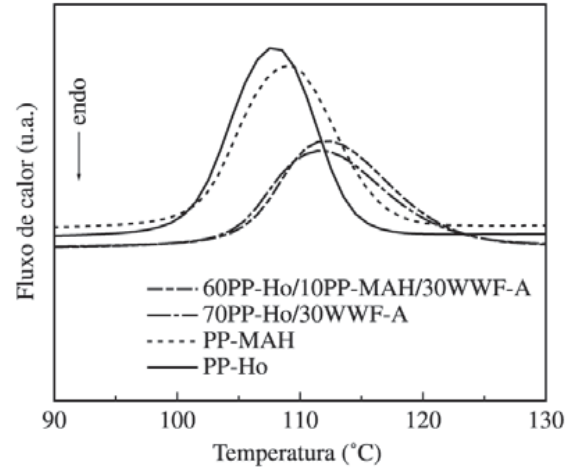

(a)

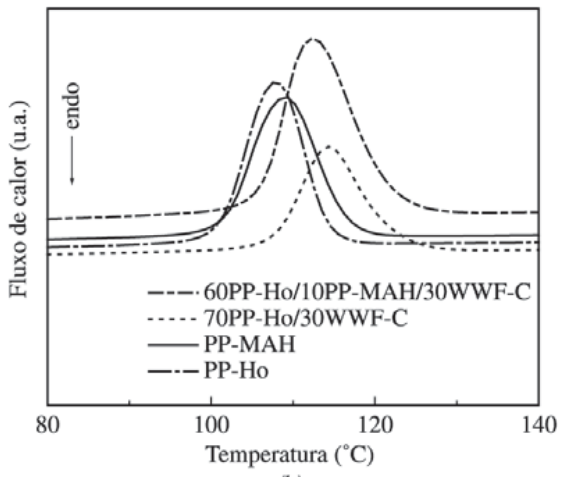

(b)

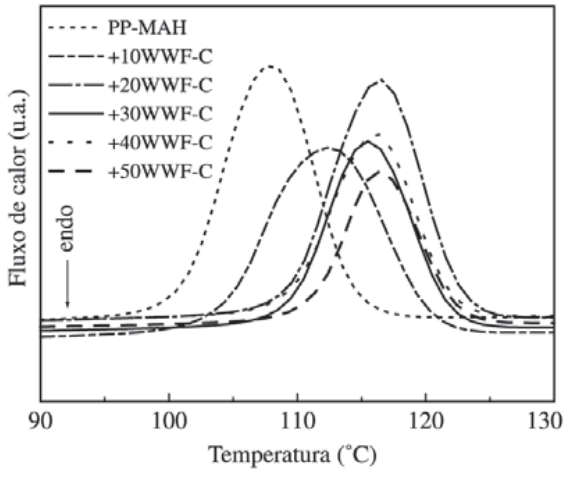

(c)

Figura 9. Temperatura de cristalização após a segunda varredura para compósitos de polipropileno com farinha de madeira. (a) WWF-A; (b) WWF-C; e (c) Efeito da concentração de farinha de madeira (WWF-C) na temperatura de cristalização do PP-MAH.

utilizando microfibra de celulose e sisal em polipropileno, mostraram que embora a temperatura de fusão permaneça praticamente constante na presença da fibra celulósica, a temperatura de cristalização, Tc da matriz termoplástica tende a se deslocar para valores mais elevados com o aumento do teor de celulose no compósito ${ }^{[23]}$.

Os resultados de DSC não apresentaram alterações significativas na temperatura de fusão do polipropileno na presença da farinha de madeira conforme observado nas curvas apresentadas na Figura 10(a) para a série completa dos compósitos estudados. Todavia, o grau de cristalinidade foi reduzido em cerca de $50 \%$ com a adição da farinha de madeira 10(b). Observou-se ainda uma queda na estabilidade térmica do sistema e maior tendência ao escurecimento da mistura com WWF-C, possivelmente devido à presença de partículas muito finas nessa composição em relação a amostra com WWF-A. Esses resultados ressaltam a importância da classificação granulométrica nas propriedades dos compósitos.

\section{Aspectos Morfológicos}

Através da microscopia eletrônica de varredura foi possível se avaliar a eficiência do processo de dispersão da carga celulósica na matriz termoplástica e a capacidade de molhamento da superfície da fibra pela resina como forma de se avaliar a eficiência do agente compatibilizante. Aspectos relacionados à molhabilidade e nível de adesão interfacial fibra-matriz podem ser observados nas micrografias apresentadas na Figura 11 para os compósitos 70PP-Ho/ PP-MAH/30WWF para dois tipos de farinha de madeira (WWF-A e WWF-C).

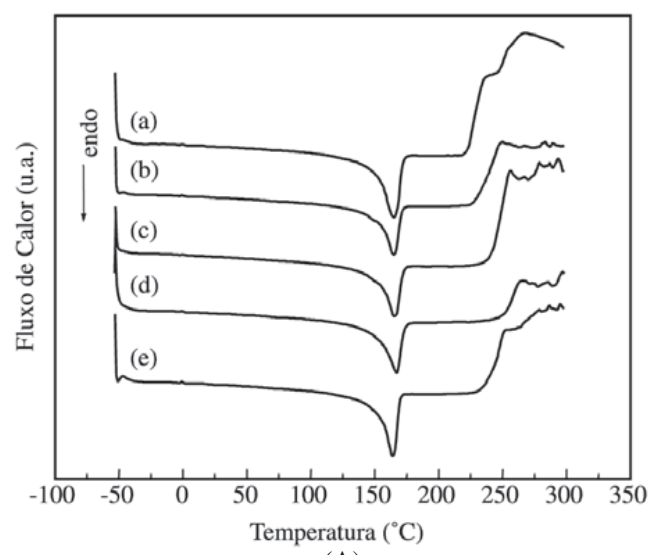

(A)

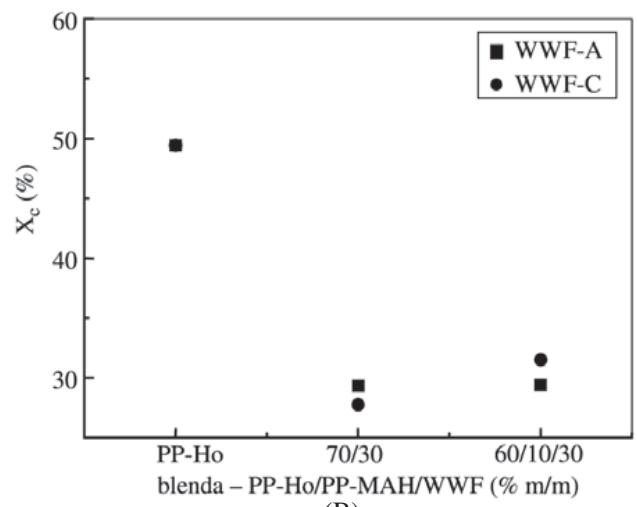

(B)

Figura 10. (A) Curvas de DSC dos materiais: (a) PP-MAH, (b) $70 \mathrm{PP}-\mathrm{Ho} / 30 \mathrm{WWF}-\mathrm{A}$, (c) $60 \mathrm{PP}-\mathrm{Ho} / 10 \mathrm{PP}-\mathrm{MAH} / 30 \mathrm{WWF}-\mathrm{C}$, (d) 70PP-Ho/30WWF-C; (e) 60PP-Ho/10PP-MAH/30WWF-C. (B) Variação do grau de cristalinidade para as misturas $\mathrm{PP}-\mathrm{Ho} / \mathrm{PP}-\mathrm{MAH} / \mathrm{WWF}$ para dois tipos de resíduos. (Taxa de aquecimento $20^{\circ} \mathrm{C} / \mathrm{min}$; dados $2^{\mathrm{a}}$ varredura). 


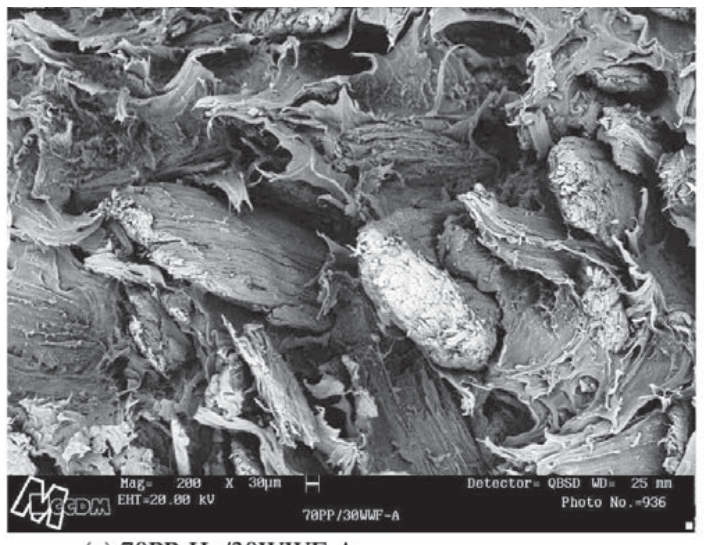

(a) $70 \mathrm{PP}-\mathrm{Ho} / 30 \mathrm{WWF}-\mathrm{A}$



(c) $60 \mathrm{PP}-\mathrm{Ho} / 10 \mathrm{PP}-\mathrm{MAH} / 30 \mathrm{WWF}-\mathrm{A}$

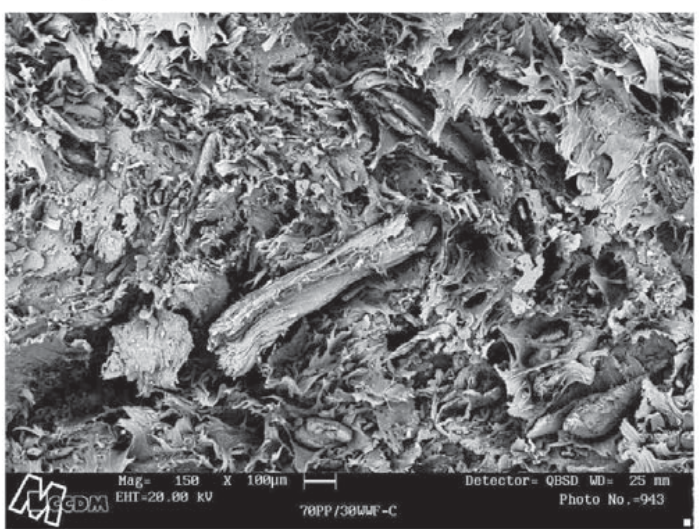

(e) 70PP-Ho/30WWF-C

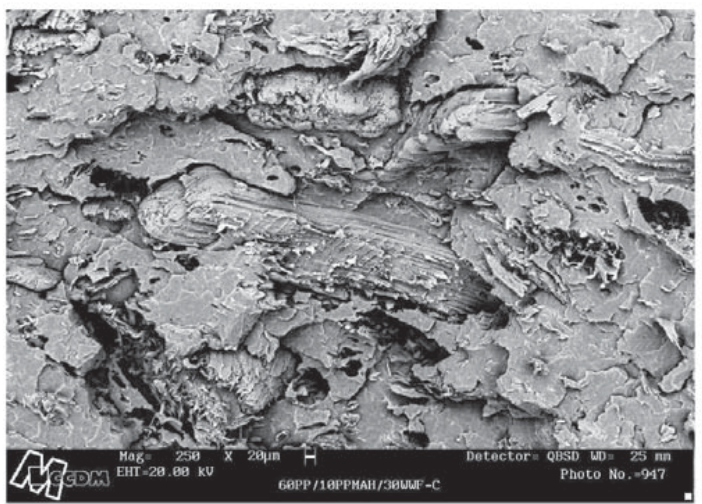

(g) 60PP-Ho/10PP-MAH/30WWF-C

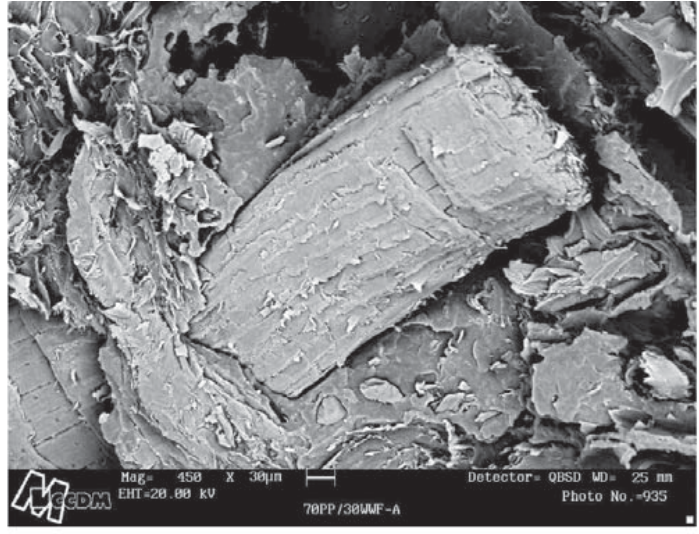

(b) 70PP-Ho/30WWF-A

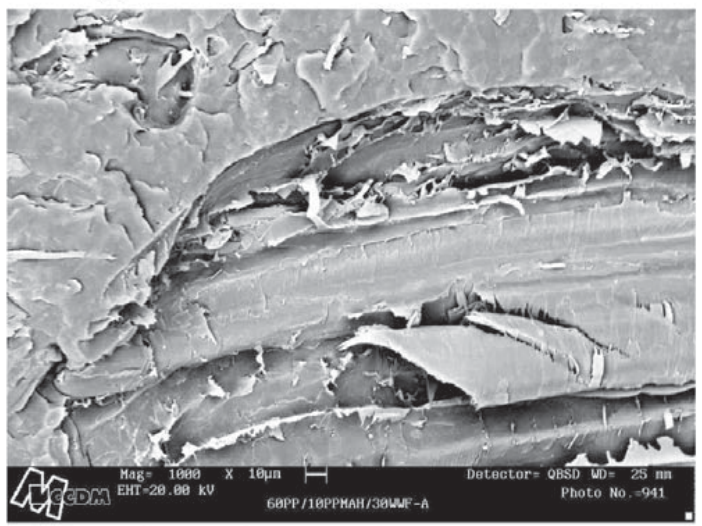

(d) 60PP-Ho/10PP-MAH/30WWF-A

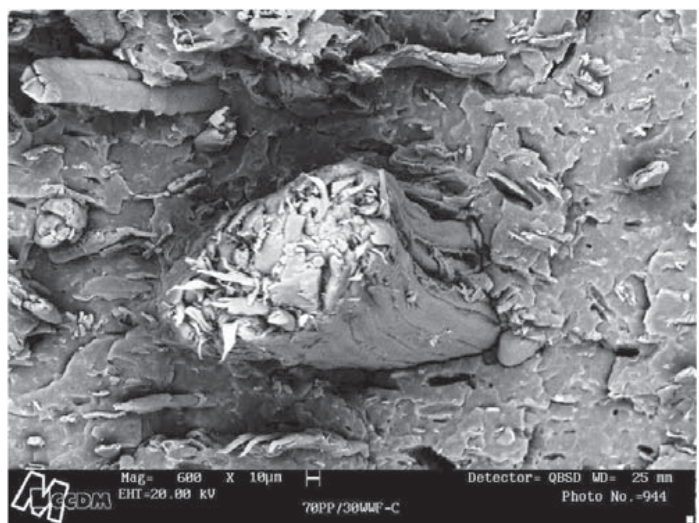

(f) 70PP-Ho/30WWF-C

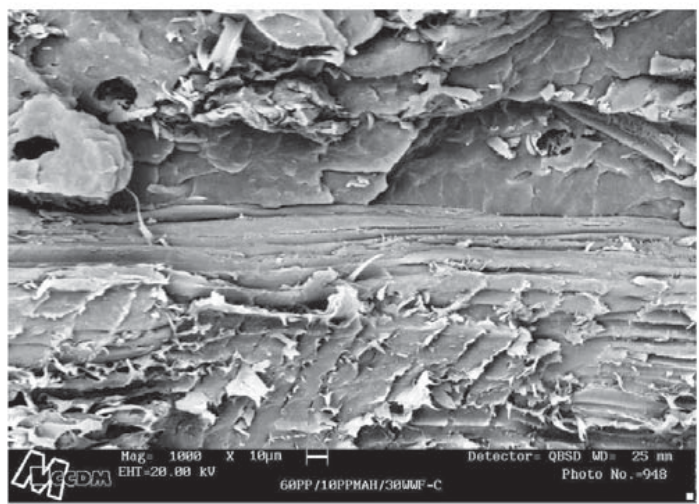

(h) 60PP-Ho/10PP-MAH/30WWF-C

Figura 11. Características morfológicas de compósitos termoplásticos de polipropileno com farinha de madeira para amostras 70PP-Ho/PP-MAH/30WWFA e C compatibilizadas e não compatibilizadas com PP-MAH. 
As imagens apresentadas mostram claramente que o compatibilizante possibilita uma melhoria da adesão interfacial entre a fibra celulósica e a matriz termoplástica independente da granulometria da farinha de madeira utilizada (Figuras 11c-d e 11g-h). As imagens corroboram os resultados obtidos nos testes mecânicos onde as amostras compatibilizadas apresentaram um incremento de resistência em relação às composições não-compatibilizadas.

\section{Conclusões}

Os compósitos termoplásticos apresentam inúmeras vantagens competitivas em relação aos compósitos convencionais de polipropileno reforçados com fibra de vidro, carbonato de cálcio e talco. Entre essas estão: Temperaturas de processamento mais baixas (economia de energia); Redução de ciclos de moldagem em produtos injetados (maior produtividade); Aumento da resistência à tração e flexão; Menor peso específico, assim uma menor quantidade de resina utilizada por componente e redução de custos de transporte por tonelada; Redução de desgastes de ferramentas (baixa abrasividade).

As evidências apresentadas nos estudos de reometria de torque realizados em nossos laboratórios indicaram que a farinha de madeira tende a reduzir a viscosidade do polipropileno, sendo acentuada pela presença do compatibilizante maleico. Os resultados dos testes de tração evidenciaram a ação positiva do compatibilizante PP-MAH através do aumento do módulo de rigidez e resistência à tração das misturas compatibilizadas de polipropileno e farinha de madeira. Os ganhos de rigidez foram significativos para quantidades de até $30 \%$ em peso de WWF na mistura sendo que as misturas não sofreram alterações significativas de propriedades após a imersão em água, mantendo assim o caráter hidrofóbico da resina no compósito. A tenacidade à fratura dos compósitos em impacto apresentaram reduções significativas na presença do compatibilizante independente da granulometria da farinha de madeira. As análises de DSC mostraram que embora ocorra reforçamento da matriz termoplástica, a cristalinidade da resina sofre queda acentuada na presença de carga celulósica. Diferenças de aspecto em relação a um maior escurecimento das misturas com a farinha de madeira não-beneficiada (WWF-C), sugerem que a presença de uma maior quantidades de partículas finas pode acelerar o processo de degradação do compósito durante o processamento.

As análises de superfícies fraturadas por microscopia eletrônica de varredura também apresentaram evidências conclusivas sobre o aumento da capacidade de molhamento fibra celulósica pela resina polimérica na presença do agente compatibilizante. A melhora na adesão interfacial evidenciada pela microscopia e o incremento nas propriedades mecânicas indicam a possível ocorrência de reações de esterificação na interface polímero-madeira, conforme sugerido pela literatura.

\section{Agradecimentos}

Os autores agradecem à empresa Pinho-Pó Ind. e Com.
Ltda pela doação da farinha de madeira beneficiada, à UFSCar, ao LCAM-USF pela infraestrutura laboratorial para realização dos ensaios de calorimetria e a FAPESP (processo 02/03909-5 e 98/14756-8).

\section{Referências Bibliográficas}

1. Eckert, C.H. "Market Opportunities for natural fibres in plastics composites", in: Proceedings of the WoodPlastic Conference, p. 87-106, Baltimore, USA (2000).

2. English, B. "Wood-Plastics Materials and Processes: A Look at Twenty Five Years of Patent Activity", in: Proceedings of the Wood-Plastic Conference, p. 6072, Baltimore, USA (2000).

3. Stark, N.; Scheneider, J.P. "Waste-Wood-Derived Fillers for Plastics”, in: General Technical Report FPL-GTR91, Forest Product Laboratory/USDA, USA, 1996.

4. Frollini, E.; Leão, A.L.; Mattoso, L.H.C. (Eds) - "Natural Polymers and Agrofiber Based Composites”, Embrapa Instrumentação Agropecuária, S.Carlos, SP, 2000.

5. Zanin, M.; Disiderá, C.; Logarezzi, A.; Correa, C.A. "Sistematização da extensão do uso de resíduos de serrarias e potencialidade de aplicação”,in: Anais do ENTAC-2002 - IX Encontro Nacional de Tecnologia do Ambiente Construído, 7 a 9 maio de 2002, Foz do Iguaçu, PR.

6. Stark, N.M.; Berger, M.J. "Effect of particle size on properties of wood-flour reinforced composites", in: Proceedings of the Fourth International Conference on Woodfiber-Plastic Composites, p. 134-143, Madison, Wisconsin, USA (1997).

7. Masson, T.J. "Desenvolvimento e reciclagem do polipropileno modificado pela presença de cargas híbridas", Tese de Doutorado, Universidade Mackenzie, São Paulo, SP (1998).

8. Machado, A.; Stuart, K. "A comparison of process configuration for compounding wood-plastics composites", in: Proceeding of the Wood-Plastic Conference, p. 73-85, Baltimore, USA (2000).

9. Markovich, N.E.; Aranguren, M.I.; Reboredo, M.M. "Some aspects related to the processing of natural fiberpolymer composites", in: Proceedings of the Natural Polymers and Composites IV, p. 416-421 São Pedro, SP. (2002).

10. Sanadi, A.R.; Caulfield, D.F.; Jacobson, R.E. in "Agrofiber thermoplastic composites, paper and composites from Agro-Based Resources". Eds. Rowell, R. M, Young, R.A.; Rowell, J.K. Cap. 12, p. 377-401, Lewis Publishers (1997).

11. Felix, J.M.; Gatenholm, P. "The Nature of adhesion in composites of modified cellulose fibers and polypropylene”. Journal of Applied Polymer Science, Vol 42, p. 609-620 (1991).

12. Youngquist, J.A.; Karmaker, A.C. "Injection Molding of Polypropylene reinforced with short jute fibers". 
Journal of Applied Polymer Science 62, p. 1147-1151 (1996).

13. Gassan, J.; Bledzki, A. "The influence of fiber-surface treatment on the mechanical properties of jutepolypropylene composites". Composites, Part A, p. 1001-1005 (1997).

14. Karnani, R.; Krishnan, M.; Narayan, R. "Bio-fiberreinforced polypropylene composites". Polymer Eng. \& Sci. 7, n. 2, p. 476-483 (1997).

15. Kazayawoko, M.; Balatinecz, J.J.; Matuana, L.M. "Surface modification and adhesion mechanisms in woodfiber-polypropylene composites”. J. Matl. Sci. 34, p. 6189-6199 (1999).

16. Wu, J.; Yu, D.; Chi-Ming, C.; Kim, J.; Mai, Y. "Effect of fiber pretreatment condition on the interfacial strenght and mechanical properties of wood fiber/PP composites". J. Appl. Polym. Sci., vol. 76, p. 1000-1010 (2000).

17. Ichazo, M.N.; Albano, C.; González, J.; Perera, R. e Candal, M.V . "Polypropylene/woodflour composites: treatments and properties”. Composite structures 54, p. 207-214 (2001).

18. Costa, T.H.S.; Carvalho, D.L.; Souza, D.C.S.; Coutinho, F.M.B.; Pinto, J.C.; Kokta, B.V. "Statistical experimental design and modelling of polypropylene-wood fiber composites", Polymer Testing 19, p. 419 (2000).

19. Coutinho, F.M.B.; Costa, T.H.S.; Carvalho, D.L.; Gorelova, M.M.; de Santa Maria, L.C. "Thermal behaviour of modified wood fibers", Polymer Testing 17, p. 299 (1998).
20. Coutinho, F.M.B.; Costa, T.H.S. “Performance of polypropylene-wood fiber composites”, Polymer Testing 18, p. 581 (1999).

21. Maiti, S.N. "Wood-flour polypropylene composites: Structure-property relationships", in: Proceedings of the Fourth International Conference on WoodfibrePlastic Composites, p. 133-144, Madison, Wisconsin, USA (1997).

22. Oksman, K.; Clemons, C. "Mechanical properties and morphology of impact modified polypropylene-wood flour composites”, J. Appl. Polym. Sci. 67, p. 1503 (1998).

23. Kuruvilla, J.; Mattoso, L.H.C. "Recent develop-ments in natural fibre based polymer composites", Natural Polymers and Composites IV, p. 443-454. Águas de São Pedro, SP (2002).

24. English, B.; Clemons, C.M. "Weight reduction: wood vs mineral fillers in polypropylene”, in: Proceedings of the Fourth International Conference on WoodfiberPlastic Composites, p. 237-244, Madison, Wisconsin, USA (1997).

25. Mano, E.B. "Polímeros como materiais de engenharia”, Ed. Edgard Blucher, Ltda, São Paulo, SP (1991).

26. Jones, F.R. "Handbook of Polymer-Fibre Composites", Longman Scientific \& Technical, Essex, Inglaterra (1994).

Recebido: 24/10/2002

Aprovado: 26/05/2003 